\title{
Model Approximate Method of Complex System
}

\author{
Juan Chen, Yuqin Liu, Shi'ang Qi, and Qing Guo
}

\begin{abstract}
During the internal model control of multivariate multiple time delay system, process model is very important in the design of controller, but complicated mathematical model is often encountered in decoupled multivariable multiple time delay system and needs to be reduced order. So this paper proposes a low order identification structure and an optimization method for model approximation of the complex model containing the time delay and non-minimum phase parts. In this paper, with the adoption of Pade approximation, a suboptimal approximate algorithm is used for the model approximation and model identification. The integral square error index and the frequency-domain integral square error index, as well as the integral time absolute error index is used to evaluate the approximate model comprehensively. Simulation results show that using the proposed model identification structure and adopting the suboptimal approximate algorithm to deal with this kind of approximation, that can get an approximate model that well reveals the dynamic characteristics of system and has a high approximation precision.
\end{abstract}

Index Terms-Model approximation, complicated model, suboptimal approximate algorithm, error performance index.

\section{INTRODUCTION}

Model identification and model approximation is often used in the design and analysis of control system, but the difficulty of controller design is determined by the complexity of the model. During the design and implementation of the multivariate multiple time delay system, mathematical model of control plant is relatively complex after decoupling [1], [2], so, model approximation is often needed to simplify the design of the controller. Model approximation causes a wide attention in the field of industrial control especially in the process of model predictive control and model optimization [3]. The purpose of model approximation can be described to find a low order approximate model that can fully describe the dynamic behavior of the complex system. The precision of the low order approximate model is of great importance since it determines whether the controller design is good or not. In the past few decades, significant research studies on model reduction have been reported, such as Krylov Subspace method [4], Pade approximation method [5], Routh

Manuscript received November 19, 2014; revised January 13, 2015. This work was supported in part by the National natural science foundation of China under Grant BS 21376014.

Juan Chen, Yuqin Liu, and Qing Guo are with the School of Information Science and Technology, Beijing University of Chemical Technology, Beijing 100029, China (e-mail: jchen@ mail.buct.edu.cn, liuyuqinbuct@163.com, guoqing@mail.buct.edu.cn).

Shi'ang Qi is with the School of Huazhong University of Science and Technology, Hubei 430074, China (e-mail:15801299065@qq.com) approximation method [6], Dominant Poles method, Continued Fraction method [7], Gradual Waveform estimation method [8], Balance order reduction method, and so on. Though all these methods are suitable for linear systems, they are not useful to non-linear system. In recent years, there are some model order reduction researches in the time delay system and the unstable system, paper [9] disused the identification method of non- equilibrium time delay model problems from closed loop step test, Dingyu Xue [10], [11] presented a Suboptimal reduction algorithm for the model order reduction of single input single output time delay system or the none time delay systems.

However, though multivariable multiple time delay systems or multivariable multiple time delay systems combined with non-minimum phase system is often encountered in the internal model control, the identification and approximate research about this model is rarely reported Although paper [12] proposed a frequency weighted recursive least squares (FRLS) algorithm solved one case of the complex model and made some achievements, but the derivation formula of the frequency-domain weighted recursive least squares algorithm is complex, besides it needs to introduce a new external loop to solve time delay parameters, the calculation of which is tedious and inconvenience in engineering application, what's more, the paper [12] did not study of the non-minimum phase system. Other papers, genetic algorithm is used for model approximation [1], but it needs large amount of calculation and optimization process may easily fall in premature, when encounters a complex problem, the computation time will be a problem that largely affecting the industrial implementation. However, the Suboptimal reduction algorithm in paper [10], [11] has the advantages of simple calculation, good delay fitting features, and can keep the stability of the original system. Hence, aiming at this problem, the suboptimal reduction algorithm is been extended to approximate the multivariable multiple time delay systems and multivariable multiple time delay systems combined with non-minimum phase system [1], [2] in this paper.

In model identification or model approximation, the main purpose is to solve the model structure and the optimization algorithm .The reasonable model structure, more simplified and effective fast optimization algorithm is the key to the model identification and model approximation. And choose a reasonable error evaluation index is the basis to evaluate whether approximate model is good or bad. Based on multivariable multiple time delay systems or multivariable multiple time delay systems combined with non-minimum phase system which is often encountered in internal model control [1], [2] This paper proposes an approximate model structure and a Suboptimal approximate method, as well as 
the error metrics quality for evaluating the model approximation performance, The approximate results are compared with frequency-domain weighted RLS method.

This paper is organized as follows. Section II presents the approximate model structure. In Section III the error metrics quality will be introduced. Section IV presents the Suboptimal approximate scheme. Then in Section V different simulation results will be discussed in this work. Finally, Section VI brings to a close of the paper.

\section{APPROXIMATE Model StruCtURE}

During the controller design of internal model system with multivariable time delay, the encountered mathematical model is often composed by two or more delay parts combined with the non-minimum phase model [1], [2] This kind of mathematical model of the system are often expressed by the following transfer function $G(s)$

$$
G(s)=G_{1}(s) e^{-\tau_{1} s} \ldots \ldots+G_{l}(s) e^{-\tau_{l} s}
$$

where $G_{l}(s)=\frac{\sum_{i=0}^{m} b_{l, i} s^{i}}{\sum_{j=0}^{n} a_{l, j} s^{j}} ;(l=1 . . k),(m<n)$ are rational transfer functions, $a_{l, j}, b_{l, i}(i=0,1, \ldots, m ; j=0,1, \ldots 2, n)$ are the coefficients of the rational transfer function, $\tau_{1} \ldots \tau_{l}$ is different time delay, Notice that when $l=2, G(s)$ is the two time delay parts superposition system, which widely exists in the internal model control.

The model structure of multivariate time delay superposition system $G(s)$ is complicated, it is hard to design its internal model controller directly, so it needs to get an approximate simplified model $G r(s)$ which has closely dynamic characteristics of $G(s)$ to simplify the design of the controller. The selection structure of simplified approximation model is directly related to the approximation effect, and it also determines the difficulty of the subsequent controller design.

The following approximate model structure is used for the above complex model:

First order plus time delay model (First order reduced model referred to as "FORM"):

$$
G r_{\mathrm{m}}(s)=\frac{k}{T s+1} e^{-\tau s}
$$

Second order plus time delay model with arbitrary poles (Second order reduced model referred to as "SORM")

$$
G r_{m}(s)=\frac{k}{s^{2}+a_{1} s+a_{0}} e^{-\tau s}
$$

The model structure in (2) is mainly used to approximate model whose unit step response is without overshoot, besides, the model structure (3) can be used to approximate model not only whose unit step response is overshoot but also whose unit step response is without overshoot.

In many cases, equation (3) can approximate the complex object model very well, and it is good enough to meet the dynamic characteristics of the original system. But when the original complex system contains non-minimum phase model structure, the model structure in (3) can not reflect the non-minimum phase characteristics of the original system, thus causes the subsequent design of controller ignore the influence of non-minimum phase, and hard to reach the ideal control effect.

Motivated by the above problems, the paper proposed a second order plus time delay model with zeros (Second order reduced non-minimum model referred to as "SORNM") presented as the following expression, for the complicated model approximation.

$$
G r(s)=\frac{b_{1} s+b_{2}}{s^{2}+a_{1} s+a_{2}} e^{-\tau s}
$$

where $a_{1}, a_{2}, b_{1}, b_{2}, \tau$ are parameters that to be identified.

\section{The ERror MEtrics QuALITY}

In this paper, the error functional integral evaluation index is used to evaluate model approximation method, the error functional integral evaluation index usually uses instantaneous error function $e(t)$ for functional integral evaluation, which involve various index such as the integral absolute error index(IAE), the integral square error index( ISE), the integral square time error index(ISTE), the integral time square error index(ITSE), and the integral time absolute error index (ITAE).The IAE performance index is suitable for evaluating the system with medium and small deviation transition process, systems designed based on this kind of performance index have the advantage of appropriate damping and a good transient response, however, the IAE performance index can not reflect obviously to the change of system parameters. The ISE performance index is suitable for process evaluation if the system transition appears large deviations. The ISTE index weighs emphasis on the late transition process error, has a good selectivity, and reflects the quickness and accuracy of the system. The ITSE performance index is focusing more on the late appear transient response error than the large initial error response. It is widely used for its good practicability and selectivity (the large the performance indicators change follows parameters change is, the better the selectivity is). Many literatures consider ITAE performance index as one of the best performance index to evaluate single input single output control systems and adaptive control systems [13]. In this paper, it is also as an index to evaluate the approximation performance of multivariable system.

The integral squared error index (ISE) and the frequency-domain integral squared error index (FISE)as well as the integral time absolute error index (ITAE) are adopted to analyze the approximation accuracy of the model.

Any order approximate model can be expressed as

$$
G r(s)=\frac{Y_{1}(s)}{R(s)}=\frac{a_{0}+a_{1} s \ldots+a_{m} s^{m}}{b_{0}+b_{1} s \ldots b_{n-1} s+s^{n}}
$$


where coefficients $\left(a_{0}, \ldots, a_{m}\right)$ and $\left(b_{0}, \ldots, b_{n-1}\right)$ represent the vector $\left(a^{T}\right)$ and $\left(b^{T}\right)$ respectively. Essentially, the model approximation is based on the set of coefficients $\left(a_{0}, \ldots, a_{m}\right)$ and $\left(b_{0}, \ldots, b_{n-1}\right)$.

Equation (5) can be written as the time domain differential equation form

$$
\begin{gathered}
y_{1}^{(n)}(t)+b_{n-1} y_{1}^{(n-1)}(t)+\ldots+b_{1} y_{1}^{(1)}(t)+b_{0} y_{1}(t) \\
=a_{m} r^{(m)}(t)+\ldots+a_{1} r^{(1)}(t)+a_{0} r(t)
\end{gathered}
$$

where $r(t)$ is the system input, $y_{1}(t)$ is the approximate model output, let $y(t)=y_{1}(t)+\Delta(t)$ as the output of the original system, $\Delta(t)$ as the output signal deviation which is caused by model approximation. Let the actual output instead of the approximate output, the 6 is equivalent to the following expression.

$$
\begin{gathered}
e(t)=y^{(n)}(t)+b_{n-1} y^{(n-1)}(t)+\ldots+b_{1} y^{(1)}(t)+b_{0} y(t) \\
-\left[a_{m} r^{(m)}(t)+\ldots+a_{1} r^{(1)}(t)+a_{0} r(t)\right]= \\
\Delta^{(n)}(t)+c_{n-1} \Delta^{(n-1)}(t)+\ldots+c_{1} \Delta^{(1)}(t)+c_{0} \Delta(t)
\end{gathered}
$$

The error $e(t)$ contains the deviation signal and the deviation of the derivative signal, which means the dynamics characteristic differences between the approximation model and the original model.

The Laplace change of the error signal is expressed as

$$
\begin{gathered}
E(s)=\left[s^{n}+b_{n-1} s^{n-1}+\ldots+b_{1} s+b_{0}\right] Y(s) \\
-\left[a_{m} s^{m}+\ldots+a_{1} s+a_{0}\right] R(s)
\end{gathered}
$$

For a particular input $r(t)$, ISE and ITAE performance index is written as :

$$
\begin{gathered}
J=\int_{0}^{\infty} e^{2}(t) d t \\
J=\int_{0}^{\infty} \mathrm{t}|e(t)| d t
\end{gathered}
$$

By using the popular Parseval theorem, from (9), gets the following frequency domain index

$$
J=\frac{1}{2 \pi j} \int_{-j \infty}^{j \infty} E(s) E(-s) d s
$$

Let $s=j \omega$, and write (9) as the discrete form index

$$
J=\sum_{i=1}^{N} v_{i}\left|R\left(j \omega_{i}\right) G\left(j \omega_{i}\right)-R\left(j \omega_{i}\right) G r\left(j \omega_{i}\right)\right|^{2}
$$

Substitute $A(s)=a_{1} s^{2}+a_{2} s+a_{3} s$ and $B(s)=b_{1} s+b_{2}$, According to (4), it gets

$$
G r(s)=\frac{b_{1} s+b_{2}}{a_{1} s^{2}+a_{2} s+a_{3}} e^{-\tau s}=\frac{B(s)}{A(s)} e^{-\tau s}
$$

Put (13) into (12), thus (13) is further written as

$$
J=\sum_{i=1}^{N} v_{i}\left|R\left(j \omega_{i}\right) G\left(j \omega_{i}\right)-R\left(j \omega_{i}\right) \frac{B\left(j \omega_{i}\right)}{A\left(j \omega_{i}\right)} e^{-\tau j \omega_{i}}\right|^{2}
$$

Noticed that (14) is the minimized function of frequency domain which is called frequency- domain integral square error [14].Take the problem in (12) and (14) into account within a chosen frequency range $\omega_{\min } \sim \omega_{\max }, \quad$ where $v_{i}=\omega_{i+1}-\omega_{i}$ (assume that $\left.\omega_{1}<\omega_{2}<\ldots<\omega_{N}\right)$ are the weights. $v_{i}$ Shows that the greater the difference between two test points frequency is, the greater the weight is.

\section{Suboptimal Approximate Algorithm}

The Suboptimal reduction algorithm is proposed in paper [10] for model order reduction of single time delay system in 1994, because of the optimization in the process used Pade approximation to deal with the time delay, the reduction algorithm is called Suboptimal reduction algorithm. By using the time weighted integral squared index as a benchmark, the objective function of the Suboptimal reduction algorithm can be evaluated as

$$
J(\sigma)=\min \left(\int_{0}^{\infty} f^{2}(t) e^{2}(t, \sigma) d t\right)
$$

where $f(t)$ is a weighting function, $e(t, \sigma)$ is the error signal. $\sigma$ is a vector by composition of undetermined coefficients, array $\sigma$ such as $\sigma^{T}=\left[a_{1}, a_{2}, \ldots . a_{i}, b_{1}, b_{2} \ldots b_{j}, \tau\right]$.

Considering the weighting function $f(t)=t^{p}, p=0,1,2, \ldots$. , the meaning of the minimum integral error criterion changes with $f(t)$ changes. When $p=0$, the minimum integral error criterion is ISE index, in one hand ISE index can reach the minimum objective function fast, in another hand, it restrains the large error transition. When $p=1$, the criterion is referred to as ISTE index, ISTE index can not only control the large deviation but also shorten the adjustment time at the same time.

The Laplace transform of error signal $e(t)$ is expressed as

$$
E(s)=\left[G(s)-G_{r}(s)\right] R(s)
$$

Let $h(t)=f(t) e(t)$, the frequency domain expression of the objective function can be evaluated from

$$
J(\sigma)=\min \left(\frac{1}{2 \pi j} \int_{-j \omega}^{j \omega} H(s) H(-s) d s\right)
$$

If the rational function $H(s)$ (the Laplace transform of $h(t)$ ) does not contain the nonlinear part, the frequency domain algorithm (Astrom1970) can be used to evaluate (17) directly. However, as the existence of the time delay that makes the problem nonlinear, Pade approximation is needed 
for the time delay. The approximated error signal is rewritten as

$$
\begin{gathered}
\tilde{E}(s)=\left[G_{1}(s) P_{k 1}\left(\tau_{1}, s\right)+\cdots+G_{l}(s) P_{k l}\left(\tau_{l}, s\right)\right. \\
\left.-G r(s) P_{k}(\tau, s)\right] R(s)
\end{gathered}
$$

where $k_{1}, . . k_{l}$ is Pade approximation order of the original model, $k$ is Pade approximation order of the approximation model.

The procedures for Suboptimal approximation algorithm is as follows.

Step 1. According to the approximate model structure, select the initial approximation model $G_{r}^{0}(s) \exp \left(-\tau_{0} s\right)$

Step 2. Use (16) to obtain an error function $E(s)$, select the appropriate $f(t)$ and calculate $H(s)$.

Step 3. Obtain the objective function value in (15).

Step 4. Use Powell recursion method to iterate one step, find better estimates $G_{r}^{1}(s) \exp \left(-\tau_{1} s\right)$.

Step 5. Set $G_{r}^{0}(s)=G_{r}^{1}(s)$ and $\tau_{0}=\tau_{1}$, update data and repeat the Step 2 until satisfactory approximate model is obtained.

\section{ILLUSTRATIVE EXAMPLE}

To test the proposed reduction approach and its applications, four examples are presented. The examples given in this paper are from paper [11] and paper [2], whose internal model control design is multivariable multiple time delay systems. Example 1 and Example 2 are the models without overshoot in paper [11], Example 3 and Example 4 are the models contain non-minimum phase.

As the original model has multiple delays, so Pade approximation is needed for the delay parts.

$$
\begin{aligned}
& \text { Example (1) : } G(s)=-\frac{0.00545 e^{-10 s}}{s^{2}+0.1394 s+0.0044}+\frac{0.010326 e^{-4 s}}{s^{2}+0.1293 s+0.0042} \\
& \text { Example (2) : } G(s)=\frac{0.3543}{(13.5 s+1)(2.61 s+1)} e^{-3.14 s} \\
& \qquad+\frac{0.5519}{(16.55 s+1)(2.55 s+1)} e^{-2.345 s}+\frac{0.3853 \times(51.6 s+1)}{(15.5 s+1)(6.6 s+1)}
\end{aligned}
$$

\begin{tabular}{|c|c|c|c|c|c|}
\hline Method & Model structure & Approximate model & FISE & ISE & ITAE \\
\hline FRLS[11] & FORM & $G r(s)=\frac{3.0549 \times 10^{-4} s+1.2915}{5.6437 s+1}$ & 0.327 & 0.9205 & 10.006 \\
\hline Suboptimal & FORM & $G r(s)=\frac{0.00566 s+0.2138}{s+0.1656} e^{-0.407 s}$ & 0.2037 & 0.4077 & 8.8126 \\
\hline FRLS[11] & SORM* $^{*}$ & $G r(s)=\frac{4.3753 s^{2}+7.06 s+1.2916}{43.5119 s^{2}+10.7588 s+1} e^{-0.36 s}$ & 0.0227 & 0.081 & 2.0348 \\
\hline Suboptimal & SORM* $^{*}$ & $G r(s)=\frac{0.02079 \times s^{2}+0.1732 s+0.01751}{s^{2}+0.2106 s+0.01356} e^{-0.0784 s}$ & 0.0116 & 0.0106 & 0.2934 \\
\hline Suboptimal & SORNM & $G r(s)=\frac{0.1772 s+0.01593}{s^{2}+0.2069 s+0.01234} e^{-0.0691 s}$ & 0.0124 & 0.0153 & 0.2394 \\
\hline
\end{tabular}

TABLE I: APPROXIMATION MODELS AND PERFORMANCE INDEXES OF EXAMPLE 1

\begin{tabular}{lccccc}
\hline \hline Method & Model structure & \multicolumn{1}{c}{ Approximate model } & FISE & ISE & ITAE \\
\hline FRLS[11] & FORM & $\operatorname{Gr}(s)=\frac{-3.6737 \times 10^{-4} s+1.2197}{21.9737 s+1} e^{-6.4 s}$ & 0.5711 & 0.8401 & 33.523 \\
Suboptimal & FORM & $\operatorname{Gr}(s)=\frac{0.0591}{s+0.0485} e^{-8.14 s}$ & 0.4081 & 0.4330 & 19.166 \\
& & & & & \\
FRLS[11] & SORM & $\operatorname{Gr}(s)=\frac{1.22}{115.0258 s^{2}+23.8051 s+1} e^{-4 s}$ & 0.0662 & 0.1781 & 6.3525 \\
Suboptimal & SORM & $\operatorname{Gr}(s)=\frac{0.0055}{s^{2}+0.1278 s+0.00452} e^{-5.45 s}$ & 0.0403 & 0.0287 & 0.5424 \\
Suboptimal & SORNM & $\operatorname{Gr}(s)=\frac{0.03609 s+0.00481}{s^{2}+0.1169 s+0.003943} e^{-5.7 s}$ & 0.0314 & 0.0134 & 0.2292 \\
& & & & & \\
\hline \hline
\end{tabular}

TABLE II: APPROXIMATION MODELS AND PERFORMANCE INDEXES OF EXAMPLE 2

As the reduction model in paper [12] of Example 2 is $\mathrm{SORM}^{*}$ (the second order with all order structure), for the facility of comparison with the FRLS method in paper [12], the paper use SORM* ${ }^{*}$ structure instead of SORM in Example 2. (In fact, the SORM structure is not suitable for Example 2)

The first-order approximate models and the second order approximate models gotten by Suboptimal approximation method and FRLS approximation method, together with the FISE, ISE and ITAE performance indices for step inputs are given in Table I and Table II. Table I is the performance index contrast of Example 1, Table II is the same for Example 2.

Note that whatever using Suboptimal approximate method or FRLS approximate method, the performance indices gotten by the second order model structure is smaller than that gotten by the first order model structure, which inflects the better approximation effect. It also can be seen that the approximation algorithm which is been used, that can get 
higher model precision for step inputs than the FRLS approximate method. Besides, when chooses proposed SORNM model structure the ITAE value is smallest, and
ITAE performance index is one of the performance evaluation indexes for control system who has a better practicability and selectivity.

TABLE III: APPROXIMATION MODELS AND PERFORMANCE INDEXES OF EXAMPLE 3

\begin{tabular}{|c|c|c|c|c|c|}
\hline Method & Model structure & Approximate model & FISE & ISE & ITAE \\
\hline FRLS[12] & SORM & 1 & 0.2394 & 0.3697 & 0.6721 \\
\hline Suboptimal & SORM & $\begin{array}{l}G r(s)=\frac{3.782 s^{2}+2.234 s+1}{0.2875} \\
G r(s)=\frac{0.7541 s+0.2875}{s^{2}+0.71 s}\end{array}$ & 0.1511 & 0.2462 & 0.3119 \\
\hline FRLS[12] & SORNM & $G r(s)=\frac{-0.3584 s+0.1846}{s^{2}+0.598 s+0.1846} e^{-2.13 s}$ & 0.0108 & 0.0946 & 0.2976 \\
\hline Suboptimal & SORNM & $G r(s)=\frac{-0.4038 s+0.1923}{s^{2}+0.6154 s+0.1923} e^{-1.7 s}$ & 0.0051 & 0.0335 & 0.0282 \\
\hline
\end{tabular}

TABLE IV: APPROXIMATION MODELS AND PERFORMANCE INDEXES OF EXAMPLE 4

\begin{tabular}{lcclll}
\hline \hline Method & Model structure & \multicolumn{1}{c}{ Approximate model } & FISE & ISE & ITAE \\
\hline FRLS[12] & SORM & $G r(s)=\frac{1}{1.5712 s^{2}+2.0765 s+1} e^{-2.09 s}$ & 0.7643 & 0.8798 & 2.0314 \\
Suboptimal & SORM & $G r(s)=\frac{0.5786}{s^{2}+1.645 s+0.5786} e^{-2 s}$ & 0.5359 & 0.1898 & 1.6529 \\
& & & & & \\
FRLS[12] & SORNM & \multirow{2}{*}{$\operatorname{Gr}(s)=\frac{-1.9761 s+1.0006}{2.3362 s^{2}+3.1254 s+1} e^{-0.31 s}$} & 0.1159 & 0.1409 & 1.2600 \\
Suboptimal & SORNM & $G r(s)=\frac{-0.8264 s+0.4144}{s^{2}+1.3040 s+0.4144} e^{-0.153 s}$ & 0.0471 & 0.0762 & 1.1908 \\
& & & & &
\end{tabular}

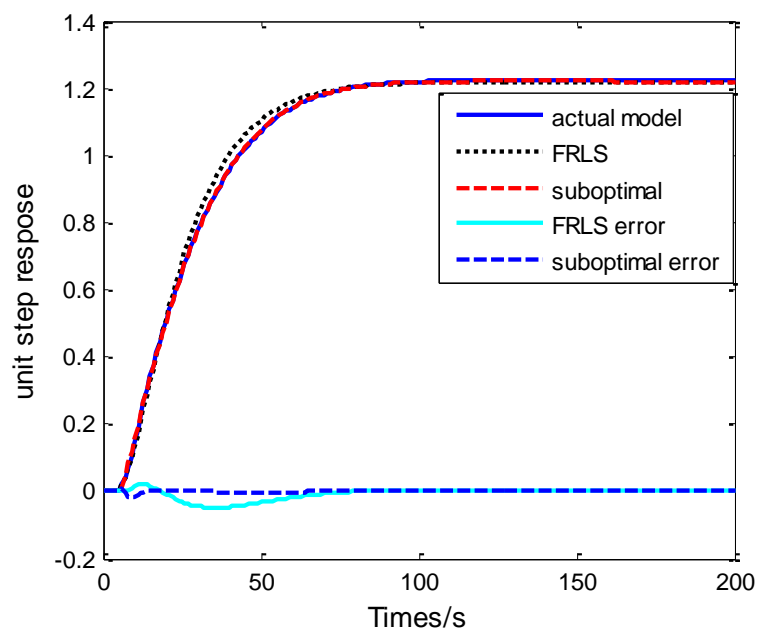

Fig. 1. Unit step responses for Example 1 and its approximate model.

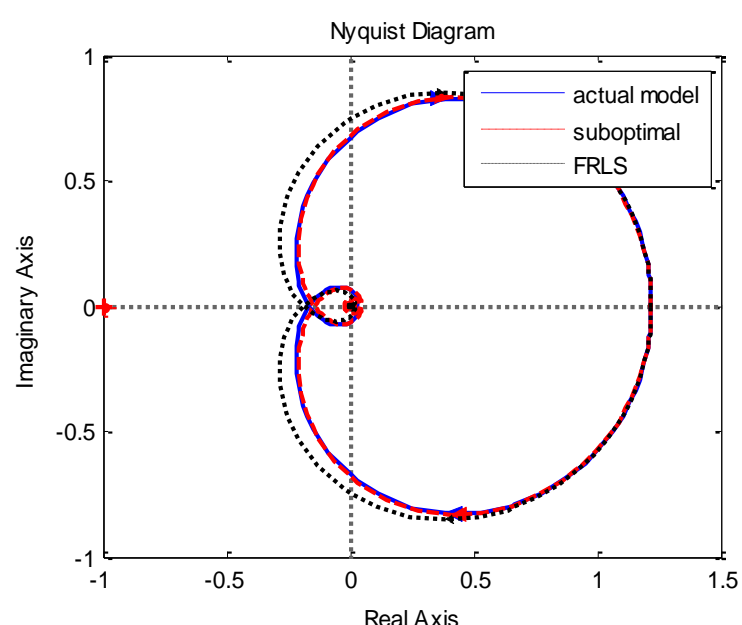

Fig. 2. Nyquist curves for Example 1 and its approximate order model.

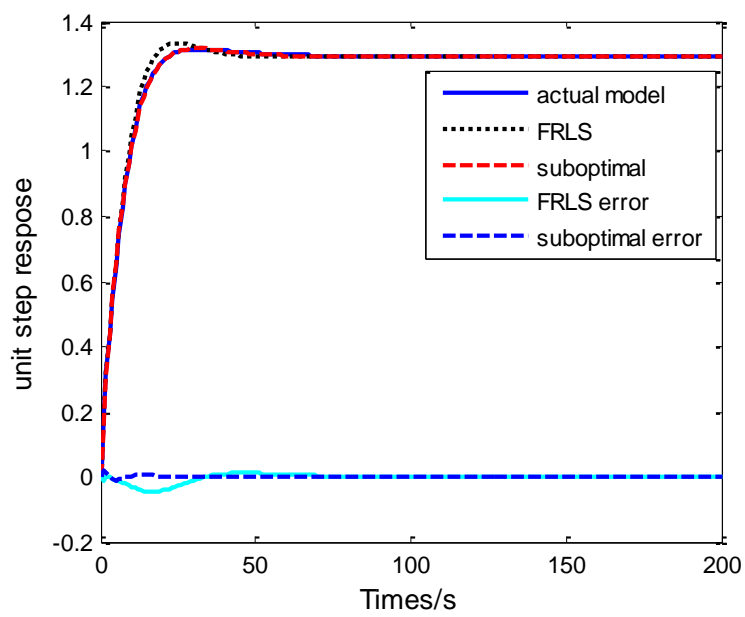

Fig. 3. Unit step responses for Example 2 and its approximate model.

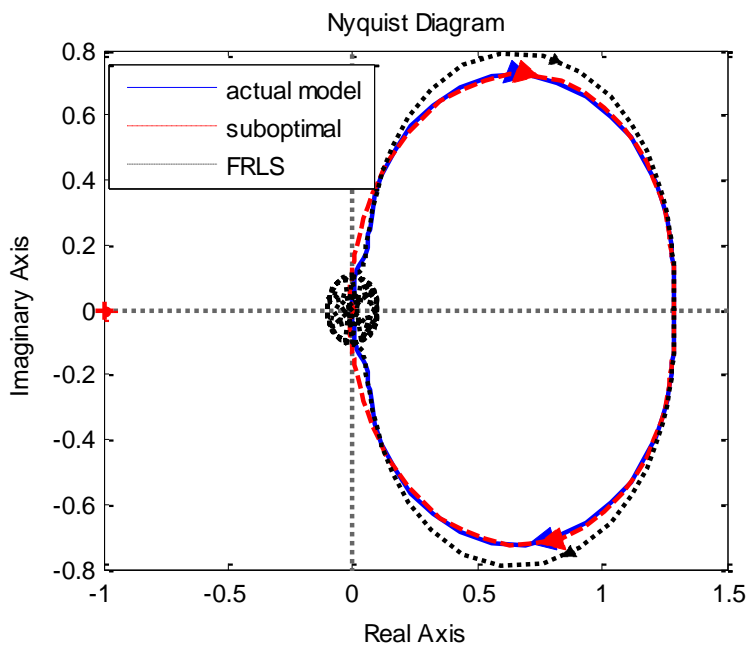

Fig. 4. Nyquist curves for Example 2 and its approximate order model. 
To further illustrate the using suboptimal algorithm and the adopting second order non-minimum phase model identification structure (SORNM) has a better effect, the unit step responses of the approximate models and the original complex model for Example 1 and Example 2 are shown in Fig. 1 and Fig. 3 for one hand, the corresponding Nyquist contrast diagrams are shown in Fig. 2 and Fig. 4 for another hand It can be seen that the suboptimal optimum model matches the original complex model extremely well over the whole time and frequency range. The FRLS models are not as good as the suboptimal optimum model especially in the frequency domain.

The original model of Example 3 and Example 4 is more complex, Example 3 is a non-minimum phase model that has unit step overshoot, and Example 4 is a non-minimum phase system with right half plane zeros. For the existence of time delay, the Nyquist diagram of original model has infinite lag with the increase of frequency, so the Pade approximation is used for the original model. The SORM approximate models and the SORNM approximate models as well as the performance criterion are shown in Table III and Table IV. It can be seen from the Table that the values of ISE index, FISE index and ITAE index gotten by SORNM structure are superior to what is gotten by SORM structure. In another words, the optimum values of the suboptimal method are better than the FRLS method.

$$
\begin{aligned}
& \text { Example (3): } G 1(s)=\frac{-0.2857}{s+2} e^{-2 s}+\frac{2.2857}{s+2} e^{-6 s} \\
& \text { Example (4): } G 2(s)=\frac{-9.1429(s-0.5)}{(s+2)^{2}} e^{-3 s}+\frac{-9.1429(s-0.5)^{2}}{2(s+2)^{3}} e^{-8 s}
\end{aligned}
$$

Fig. 5-Fig. 8 are the simulation comparisons of SORNM model and SORN model using suboptimal optimum algorithm. As can be seen from the Fig. 5 and Fig. 7, for non-minimum phase system, the use of SORN model structure ignores the right half plane zeros parts, the unit step response error is very large within the non-minimum phase range. And its frequency dynamic characteristics are comparatively large difference when compared with the original system (Fig. 6 and Fig. 8). However, the SORNM model structure joined the right half plane zeros parts, both the unit step response curve and the frequency response curves fit the original system very well.

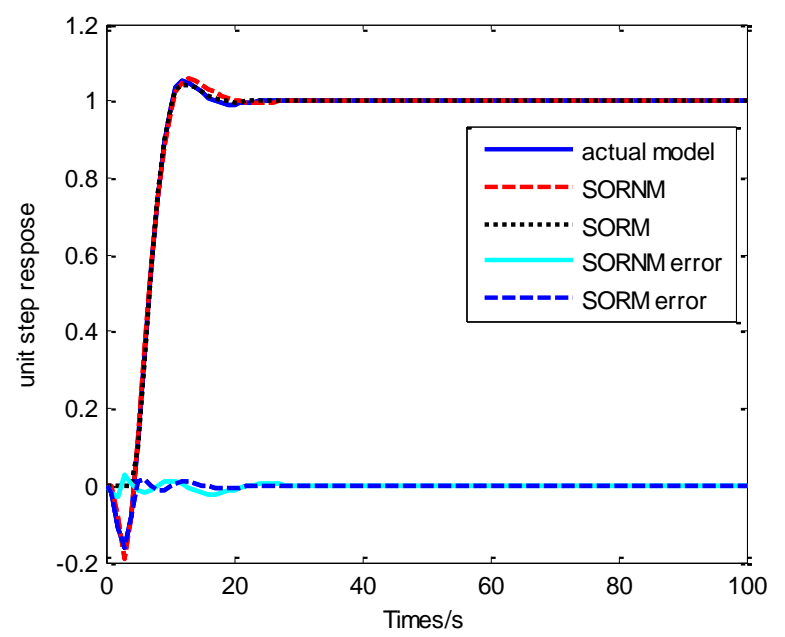

Fig. 5. Unit step responses for Example 3 and its approximate order model.

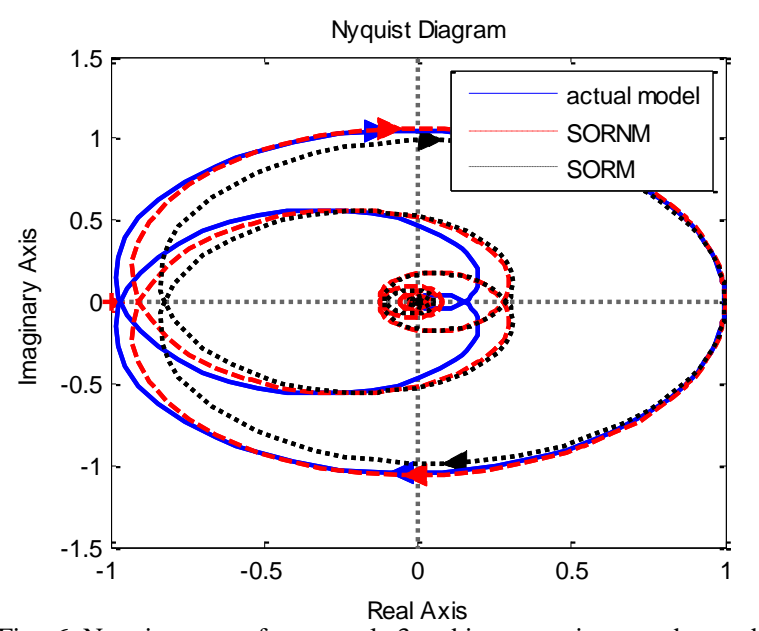

Fig. 6. Nyquist curves for example 3 and its approximate order model.

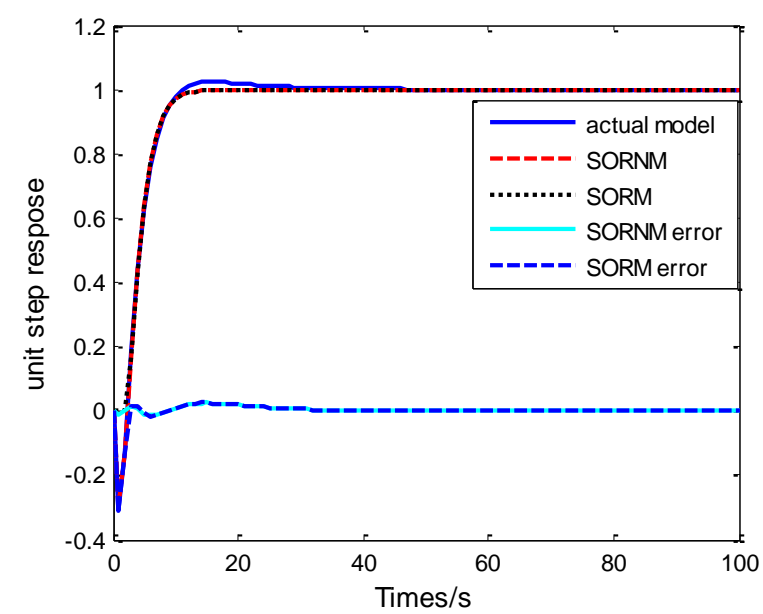

Fig. 7. Unit step responses for Example 4 and its approximate order model.

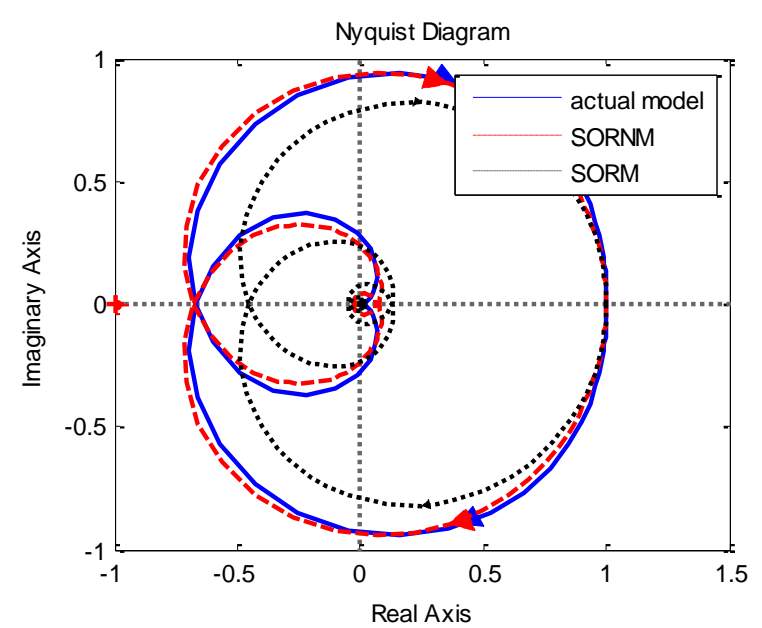

Fig. 8. Nyquist curves for Example 4 and its approximate order model.

\section{CONCLUSION}

In this paper, a SORNM identification structure has been proposed and a suboptimal approximate algorithm is used for model approximation of multivariable multiple time delay non-minimum phase system in internal model control design. The values of ITAE performance index, ISE performance index, and FISE performance index are been used to evaluate the approximation result. Several examples which including unit step response model with overshoot, or without overshoot , and non-minimum phase model have been given to show the effectiveness and practicability of the method, 
which adopts suboptimal approximate algorithm together with the proposed SORNM identification structure. The simulation results show that the approximate resultant model gives a higher precision to the unit step response and frequency response of the original model compared with FRLS algorithm

\section{REFERENCES}

[1] J. Chen, Z. He, and X. Qi, "A new control method for MIMO first order time delay non-square systems," Journal of Process Control, vol. 21, pp. 538-546, 2011.

[2] G. Min, J. Chen, and P. Yawei, "The control method of multivariable time delay square system containing right half plane zeros," Procedia Engineering, vol. 15, pp. 1004-1009, 2011.

[3] Q. G. Wang and Y. Zhang, "Fast algorithm for reduced-order modeling," ISA Transactions, vol. 38, pp. 225-230, 1999.

[4] V. Druskin and V. Simoncini, "Adaptive rational Krylov subspaces for large-scale dynamical systems," Systems and Control Letters, vol. 60, pp. 546-560, 2011.

[5] R. W. Jones, "Recursive estimation of time delay using a high-order pade approximant," in Proc. E Annual Conference, 2004, pp. $1328-1333$.

[6] R. Prasad, "Pade type model order reduction for multivariable systems using Routh approximation," Computers and Electrical Engineering, vol. 26, pp. 445-459, 2000.

[7] T. Y. Guo, C. Hwang, and L. S. Shieh, "Model reduction of nonsquare linear MIMO systems using multipoint matrix continued-fraction expansions," Journal of the Franklin Institute, vol. 331, pp. 189-216, 1994.

[8] J. M. Wang, C. C. Chu, and Q. J. Yu, "On projection-based algorithms for model-order reduction of interconnects," IEEE Transactions on Circuits and Systems, vol. 49, pp. 1563-1585, 2002.

[9] T. Liu and F. Gao, "Closed-loop step response identification of integrating and unstable processes," Chemical engineering science, 2010, vol. 65, no. 10, pp. 2884-2895.

[10] D. Y. Xue and D. P. Atherton, "A suboptimal reduction algorithm for linear systems with a time delay," International Journal of Control, vol. 60, pp. 181-196, 1994.
[11] D. Xue, Computer Aided Control Systems Design Using Matlab Language Third Edition; Beijin: Tsinghua University Press, 2006, pp. 127-130.

[12] P. Zhou, T. Chai, and Q. Liu, "Frequency-domain weighted RLS model reduction for complex SISO linear system," in Proc. the American Control Conference, pp. 5719-5724, 2009.

[13] Z. Xu, H. Dong Li, and Y. Xue, "Based on ITAE index of the comparative study of PID parameter setting methods," in Proc. the CESS, vol. 23, pp. 206-210, 2003.

[14] X. Wang and H. Shao, "New method of frequency domain identification and model reduction based on Pade approximation," Control Theory and Application, vol. 21, pp. 54-58, 2003.

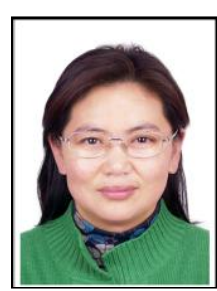

J. Chen was born in Heilongjiang Province, China, in June 1961. She is a professor of engineering in Beijing University of Chemical Technology since 2000 She is a reviewer of Journal of Process Control. Her specialization ranges from advanced control theory and control method, system identification and modeling, ultrasonic testing technology, intelligent detection technology and intelligent control. Her current research interest is in advanced control methods, intelligent detection and control.

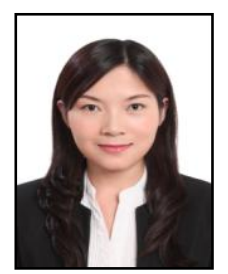

Y. Q. Liu was born in Hubei Province, China, in 1991 She obtained her bachelor's degree in automation in 2012. She is a master graduate student of Beijing University of Chemical Technology. Now She is doing her research in model identification of closed-loop system. 